\title{
The flare-up phenomenon: recurrence of distant dermatitis during patch testing
}

Aleksander Obtułowicz, Magdalena Nowak-Ślusarczyk, Magdalena Pirowska, Sylwia Lipko-Godlewska, Anna Wojas-Pelc

Department of Dermatology, Jagiellonian University Medical College, Krakow, Poland

Adv Dermatol Allergol 2016; XXXIII (1): 68-69

DOI: 10.5114/pdia.2016.57765

Irritant contact dermatitis (ICD) is a common skin condition and a risk factor for allergic contact dermatitis. The prevalence of this disease in the general population varies between $1.7 \%$ and $6.3 \%$ and is increasing. The role of the patch test in detection of contact sensitivity is of prime importance. Patch tests should be used to identify topical agents that may be responsible for exacerbation

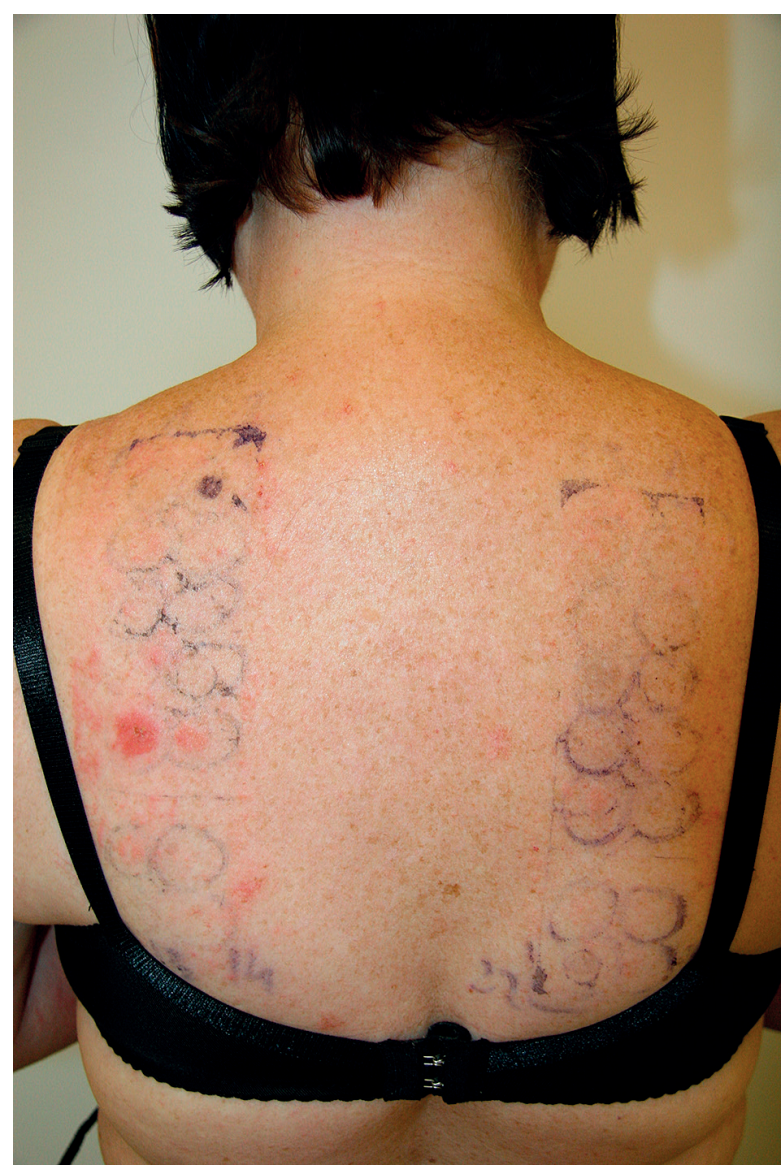

Figure 1. Positive results of the patch test or aggravation of eczema, especially in patients who do not improve despite adequate treatment.

A 53-year-old woman suffering from recurrent, longlasting dispersed eczematous skin lesions, mainly on her face and hands, was admitted to the Allergy Unit of the Dermatology Department of Jagiellonian University Medical College in Krakow for treatment, extended diagnostics and finding the reason for her illness. She was generally in a good physical condition and not taking any drugs. Skin symptoms were accompanied by an intensive itch and unrelated to work. After the discontinuation of treatment and after 2 months of full remission of skin lesions, patch tests were performed, using European standard (Chemotechnique Diagnostics) series.

A positive, erythemato-papular reaction to both colophony and parabens, corresponding to “++", was observed after 48 h. After 96 h, a strong intensification of the reaction was found only in the test site for colophony. Additionally, the reaction was accompanied by a local oedema with blisters and estimated as "+++". In the test site for parabens, the 48-hour reaction was maintained (Figure 1).

Between the first and second test readings, eczematous lesions with faint borders, growing gradually but quickly, appeared on the face and hands of the patient (Figure 2).

On the basis of the symptoms of acute allergic contact dermatitis, the flare-up phenomenon was suspected.

Systemic and topical corticosteroids were prescribed. The initial dose of steroids (prednisone) was $30 \mathrm{mg}$ a day. After $24 \mathrm{~h}$, in view of a clear, sustained improvement of the local state, the dose of prednisone was gradually reduced and finally the drug was stopped after 10 days. The appearance of multifocal eczematous lesions during the testing was considered a hardly predictable complication in the course of a standard diagnostic procedure.

The flare-up dermatitis was first time described in 1981, in a patient with nickel contact eczema [1]. In the

Address for correspondence: Magdalena Pirowska MD, PhD, Department of Dermatology, Jagiellonian University Medical College, 8 Skawinska St, 30-066 Krakow, Poland, phone: +48 600858 891, e-mail: pirowska@gmail.com Received: 15.06.2014, accepted: 16.03.2015. 


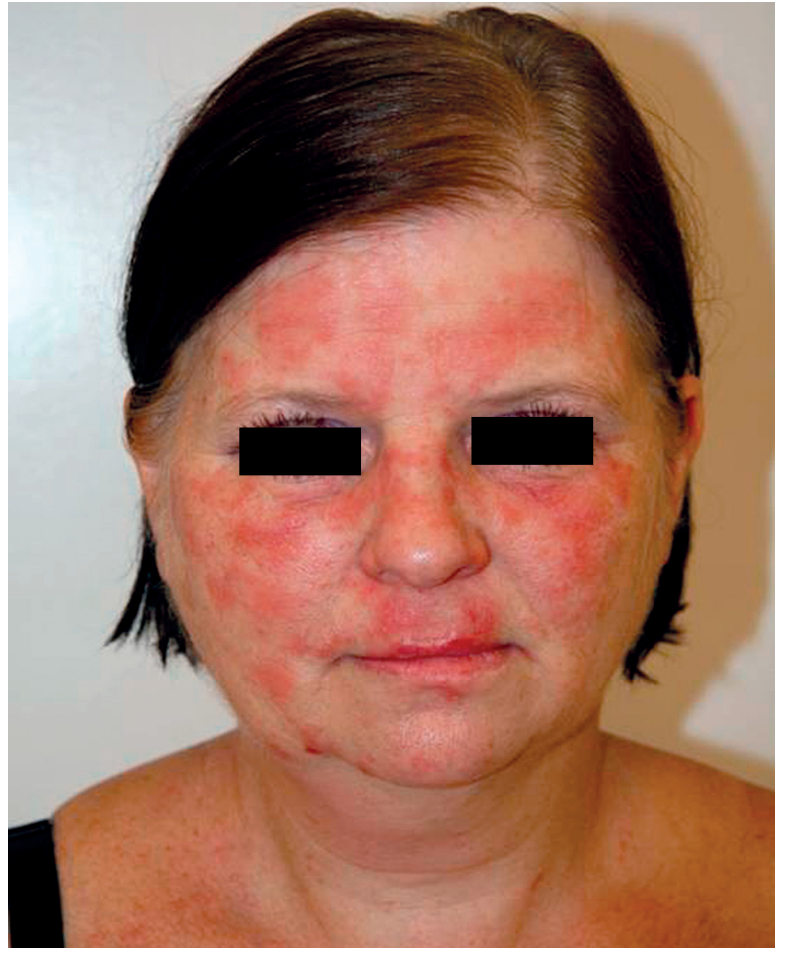

Figure 2. Erythemato-papular-oedematous rash localized on face

daily practice, patch tests remain a basic diagnostic procedure for distinguishing allergic contact dermatitis from irritant contact dermatitis. Their sensitivity and specificity reaches $70 \%$ [2]. Although the safety of patch tests is proven, we have to remember the risk of complications in some cases [1-3].

In the patient, we confirmed an allergic contact dermatitis for colophony and parabens, triggering the flareup reactions of distant dermatitis. The flare-up reactions consist of the reactivation of a previous dermatitis following the patch testing [4]. It has been frequently described as related to nickel and also in patients with multiple contact allergies - differently from our patient [1, 5].

The pathogenesis of the flare-up phenomenon during the patch testing is not completely understood. Probably it is a result of a reactivation of specific memory $T$ cells that remained in the previous area of contact $[1,6]$. On the basis of the clinical picture, history and the results of patch testing, the colophony was suspected to be the main reason for the flare-up reaction, because of the strong reaction to it in the patch testing and because of its cross-reactivity with many environmental substances in our daily life. A contact allergy to cosmetics used by our patient was suspected, as the colophony may provoke cross-reactions with many substances often present in cosmetics, like the Balsam of Peru, resins or dihydroabietyl alcohol [7, 8].

The presented case reminds us that side effects may appear during patch testing, even though this method is considered to be very safe [1]. Therefore, it is necessary to perform these tests in medical centres with well-trained personnel, with the patient supervision available during the whole testing time.

\section{Conflict of interest}

The authors declare no conflict of interest.

\section{References}

1. Reig Rincon de Arellano I, Villalon Garcia AL, Cimarra Alvarez- Lovell M, et al. Flare up to betalactams. Allergol Immunopathol 2005; 33: 282-4.

2. Spiewak R. Patch Testing for contact allergy and allergic contact dermatitis. Open Allergy J 2008; 1: 42-51.

3. Castelain M. Side effects, complications and contraindications of patch-tests. Ann Dermatol Venereol 2009; 136: 645-9.

4. Mooller H, Ohlsson K, Linder C, et al. The flare-up reactions after systemic provocation in contact allergy to nickel an gold. Contact Dermatitis 1999; 40: 200-4.

5. Mose AP, Steenfeldt N, Andersen KE. Flare-up of dermatitis following patch testing is more common in polysensitized patients. Contact Dermatitis 2012; 63: 289-90.

6. Torchia D, Capretti C, Pizzo B, et al. Patch test triggering recurrence of distant dermatitis: the flare-up phenomenon. CMAJ 2008; 12: 341.

7. Downs AM, Sansom JE. Colophony allergy: a review. Contact Dermatitis 1999; 41: 305-10

8. Paulsen E, Andersen KE. Colophonium and Compositae mix as markers of fragrance allergy: cross-reactivity between fragrance terpenes, colophonium and Compositae plant extracts. Contact Dermatitis 2005; 53: 285-91. 